\title{
Covariance Study to Evaluate the Influence of Optical Follow-up Strategies on Estimated Orbital Parameters
}

\author{
E. Cordelli*, Dr. A. Vananti, Prof. Dr. T. Schildknecht \\ Astronomical Institute of the University of Bern \\ Sidlerstrasse 5, CH-3012 Bern, Switzerland
}

\begin{abstract}
An in-depth study, using simulations and covariance analysis, is performed to identify the optimal sequence of observations to obtain the most accurate orbit propagation. The accuracy of the results of an orbit determination/improvement process depends on: tracklet length, number of observations, type of orbit, astrometric error, time interval between tracklets and observation geometry. The latter depends on the position of the object along its orbit and the location of the observing station. This covariance analysis aims to optimize the observation strategy taking into account the influence of the orbit shape, of the relative object-observer geometry and the interval between observations.
\end{abstract}

Keywords: Space debris, orbit determination, covariance, optical measurements, GEO, GTO.

\section{Introduction}

At the moment there are more than 29000 objects with a diameter bigger than 10 $\mathrm{cm}$, and more than 670000 objects with a diameter bigger than $1 \mathrm{~cm}$ in the space around the Earth; furthermore, according to estimates there are more than 170 million objects bigger than $1 \mathrm{~mm}$ [1]. Among all these objects, only about 1400 are active satellites, all the rest is space debris. Space debris is any man made orbiting object which is not operational with no reasonable expectation of assuming or resuming its intended function [2]. The space debris population includes discarded satellites, rocket bodies, mission-related objects, painting and insulation

*Corresponding author, Tel. +41 (0)31 6318819

Email address: emiliano.cordelli@aiub.unibe.ch (E. Cordelli) 
flakes, fragments created by collisions, and break up events [3]. Space debris constitutes a serious problem for space missions, both humans and satellites. In fact, in the Low Earth Orbit region (LEO) for example, the average speed for debris is about $8 \mathrm{~km}$ per second [4]; while in the Geostationary region (GEO) the average speed for debris is more than $3 \mathrm{~km}$. Because of the high velocities of the debris particles, the present shields, including those used on-board of the International Space Station (ISS), are able to protect spacecraft only from the smaller debris (less than $1 \mathrm{~cm}$ in size [3]). For these reasons the space debris is an important topic for the various space agencies and institutions, which are conducting a lot of research to better understand this problem. The Astronomical Institute of the University of Bern (AIUB) is also involved in this field of research. The most common unanswered questions are: how many debris objects are there? What are the most populated regions? What are they made of? And how will this population evolve in the future? To answer these questions the most common approach consists of three main steps: the first is the discovery of the objects [5], the second is the orbit determination [6], [7] and the third is the characterization of the objects [8], [9]. The AIUB performs all these activities using its telescopes. As mentioned before, the first orbit determination and the orbit improvement are fundamental steps for the study of space debris. The first one is performed by scanning certain regions of the sky chosen in a way to ensure that an object is observed several times during the same night [10]. The second is performed by planning regular observations of the object of interest, these additional series of observations are usually called follow-ups. Due to the huge amount of space debris and to the limitations of the telescopes, which can operate only when the weather conditions are good, it is necessary to optimize the time available for follow-up observations.

This paper will describe a method, based on the analysis of the covariance matrix, to understand how the follow-up observations should be distributed to obtain the best orbit; in addition this method can also be applied to optimize the survey strategies. This method will not provide a general rule on how to distribute observations but the idea is to suggest which observation strategy adopt having an a priori knowledge of the orbit. Thus, in the case of follow-up one already knows, even roughly, the orbit of the interested object and the method will tell where to observe to improve certain parameters (according to the user needs). In the survey case, the user already knows which kind of orbital class wants to investigate, thus the proposed method will provide information about which orbital regions to observe in order to obtain the best initial orbit. In this paper we will focus mainly on high altitude orbital regions because, especially the GEO regions, they have a high 
concentration of space debris and can be observed mainly using optical sensors. We define the best orbit as the set of orbital elements with the lowest uncertainties, that maximizes the accuracy of the predicted positions of the object. In the first part of the paper we describe the reasons that brought us to use the covariance matrix for this study and we will show the results of a theoretical study carried out to identify the main parameters which influence the results and how the problem can be simplified. Then the results obtained from a simplified scenario will be shown. Afterwards the complexity of the scenario is increased step by step showing in details the consequences on the results. Finally, this paper will present the results obtained from the application of the covariance study to some typical observation scenarios.

\section{Theory}

At the end of an observation night is not unusual to have an average of two tracklets per observed objects. A tracklet is the result of a series of images acquired during a survey campaign or during a follow-up of an already cataloged object. We assume that a standard tracklet is consisting of e.g. 7 images, each one of them contains a triplet of measurements, two angular, one in Right Ascension and one in Declination (respectively RA and DE), and a time epoch. This means that on average, from an observations night, one has 28 angular observations and 14 epochs for each object. Of course, these numbers can vary depending on factors as: the number of objects to observe in the catalog, the survey strategy and also, the performance of the software used to extract the measurements from the images. For this study we assumed to have two good tracklets per observed object. These two series of observations are then used to determine/improve the orbit of the object by mean of a Least SQuares adjustment (LSQ). The aim of this study is to analyze the output covariance of a LSQ process to understand how the geometry between observer and target object influences the accuracy of the estimated parameters. This analysis is carried out in order to be able to optimize the parameter estimation and to find the best combination of tracklets which gives the best orbit.

\subsection{What is the covariance}

The covariance matrix was chosen as the evaluation criterion because, as one can see from Eq. (1), it contains the uncertainties of the estimated parameters only as a function of the partial derivatives of the observations w.r.t. them; or rather it contains the partial derivatives of RA and DE w.r.t. semi-major axis, eccentricity, 
inclination, right ascension of ascending node, argument of perigee and the $6^{\text {th }}$ parameter, see Eq. (2). These partial derivatives are functions of the geometric relation between observer and observed object.

$$
P=m^{2}\left[A^{T} W A\right]^{-1},
$$

in which:

$$
A=\frac{\mathrm{d} o b s_{i}}{\mathrm{~d} X_{o}}
$$

where:

- $P$ is the covariance matrix,

- $m^{2}$ is the a posteriori variance factor [11],

- $A$ is the first design matrix,

- $W$ is the weight matrix.

- $o b s_{i}=\left[R A_{i} ; D E_{i}\right]$ are the $i^{t h}$ angular measurements, respectively Right Ascension and Declination, where $i=1, \ldots, n$ and $n$ is the number of observations,

- $X_{o}=\left[a, e, i, \Omega, \omega, 6^{t h}\right]$ are the orbital parameters.

The a posteriori variance factor is function of the residuals of the observations in RA and DE. In reality the residuals are function of the astrometric error on the measurements and can be also due to deficiencies in the mathematical model used in the LSQ. This quantity is not taken into account in this study because our aim is not to solve the parameters estimation problem but is only to evaluate the influence of the object-observer relative geometry on their accuracy.

To carry out this study it was assumed that the observations are performed from the same telescope system and then we don't have to address the problem of measurements with different qualities [11], [12]. This assumption allow us to make a further simplification regarding the weight matrix $(W)$ that in this case is a unit matrix of size $2 n$. Another important information is contained within the correlation indices which can be retrieved from the covariance matrix as shown in Eq. (3). These indices are useful because they tell us how strong any two parameters are correlated. 


$$
\rho_{i j}=\frac{\sigma_{i j}}{\sigma_{i} \sigma_{j}}
$$

where:

$--1 \leq \rho \leq 1$ is the correlation index,

- $\sigma_{i j}$ is covariance of the elements $i$ and $j$,

- $\sigma_{i}$ and $\sigma_{j}$ are the standard deviations of the elements $i$ and $j$.

\subsection{Choice of parameters}

As can be seen from Eq. (1) and (2), the output covariance of a LSQ adjustment is a [6x6] matrix function of the observer position and the orbit of the object. To simplify the problem, in order to isolate the contribution of each single component, it was decided to eliminate the effects associated to the observer, so in a first step an observer on the center of the Earth is used. The set of unknown parameters consists of: two parameters which describe the shape of the orbit (i.e. semi-major axis and eccentricity), two which describe the orientation of the orbital plane in space (i.e. inclination and right ascension of ascending node), one parameter for the orientation of the orbit in the plane (i.e. argument of perigee), and finally the $6^{\text {th }}$ parameter which relates the position of the object along the orbit at a particular time. Among them, the parameters describing the orientation of the plane in the space are completely independent from the others, so negligible in first analysis. The remaining parameters can not be excluded a priori because they depend on the chosen parametrization. There are several variables which can be used to express the $6^{\text {th }}$ parameter: mean anomaly, eccentric anomaly, true anomaly, argument of latitude and perigee passing time, respectively $M, E, v, u_{0}$ and $T_{0}$. Among them, the historical one is the perigee passing time; in fact, using $T_{0}$, the dependencies between parameters have the structure described by Eq. (4) [11].

$$
\begin{aligned}
M & =M\left(a, T_{0}\right) \\
E & =E(\sigma, e) \\
v & =v(e, E)
\end{aligned}
$$

Making use of this structure the partial derivatives of true anomaly are function of $a, e$ and $T_{0}$. This parametrization has a big disadvantage for small eccentricity 
values; in fact if $e=0$, the orbit is circular, which means that we do not have any perigee and the $T_{0}$ can not be defined anymore. To overcome this problem, the most used solution is to define the argument of latitude at the time $t_{0}$. For circular orbit, exploiting the definition of osculating elements [11], the user can set up arbitrarily an osculating time $T_{\text {osc }}$, with a certain $\omega$ and $v_{0}$, and solve the LSQ adjustment at this epoch. The disadvantage is given by the fact that the minimum number of parameters that one can estimate increases. In fact, according to the new parametrization shown by Eq. (5) and with the consequent dependencies between parameters the minimum number of parameters to estimate rises from 3 $\left(a, e\right.$ and $\left.T_{0}\right)$ to $4\left(a, e, \omega\right.$ and $\left.u_{0}\right)$. The analysis reported in this paper were carried out using the parametrization with the argument of latitude because of the above mentioned reasons.

$$
\begin{aligned}
v_{0} & =v_{0}\left(u_{0}, \omega\right) \\
E_{0} & =E_{0}\left(v_{0}, e\right) \\
T_{0} & =T_{0}\left(a, e, E_{0}\right)
\end{aligned}
$$

\subsection{Study of derivatives}

The study of the partial derivatives was carried out to have an idea of their influence on the results. Figure 1 shows the behavior of the partial derivatives of RA and DE w.r.t. the orbital elements, obtained for an object whose orbit is characterized by the following elements: $a=42164.173 \mathrm{~km}, e=0.6, i=30^{\circ}, \Omega=40^{\circ}$, $\omega=60^{\circ}$ and $T_{\text {osc }}=0 \mathrm{sec}$. The observer's coordinate used are: latitude $40^{\circ}$ North, longitude $10^{\circ}$ East and altitude $900 \mathrm{~m}$.

As can be seen in Figure 1, all partial derivatives show periodic features over the orbital period, which in this case is also coincident with the sidereal day. There is only an exception for the derivatives w.r.t. the semi-major axis that show also

a time dependency which amplifies the periodic features. Looking at the analytical expression of these derivatives, it was noticed that these are the only ones which are proportional to the difference between the actual epoch and the osculating time [see Eq. (6)]. During the analysis of the covariance matrix, the influence of different $T_{o s c}$ was also studied; the variations caused by $T_{o s c}$ are seen only in the uncertainties of the argument of latitude $\left(u_{0}\right)$ without influencing the other parameters.

$$
\left(\frac{\mathrm{d} R A}{\mathrm{~d} a}, \frac{\mathrm{d} D E}{\mathrm{~d} a}\right) \propto\left(t-T_{o s c}\right)
$$



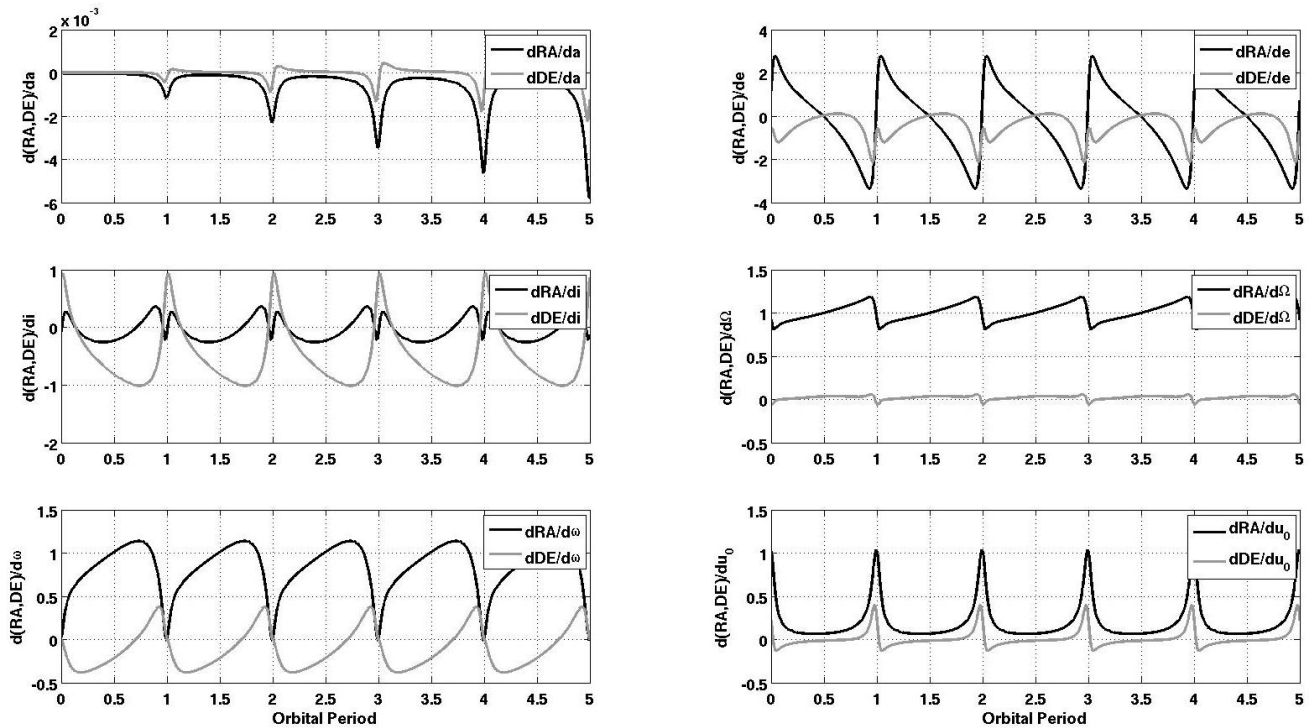

Figure 1: Behavior of RA and DE partial derivatives w.r.t. orbital elements.

\section{First Results}

The main aim of this study is to understand how the relative object-observer geometry influences the accuracy of the estimated parameters. The results which we are going to present are based on a limited number of observations, in particular only two tracklets are used, each one is made by 7 triplets of measurements (i.e. RA, DE and time epoch), for a total number of 28 angular observations and 14 epochs. During all simulations the time interval between the measurements within a tracklet is kept constant to 30 seconds. The simulations are performed in order to cover all possible combinations of tracklets positions in the orbit. To do so, the first LSQ adjustment is performed positioning the first tracklet on the orbit perigee and the time interval between first and second tracklet is increased from $10 \mathrm{sec}$ to two orbital revolutions; then, the same procedure is repeated positioning in each run the first tracklet slightly forward along the orbit. For each couple of tracklets the last iteration of a LSQ adjustment is simulated and from the relative covariance matrix, without taking into account the a posteriori variance factor, the square root of the terms in the main diagonal and the correlation coefficients are considered. Since we are not interested in LSQ performances the correct orbital elements are given as input to the LSQ. It is possible to do this simplification because being a non-linear LSQ, the only requirement to find a minimum is that the initial value of the estimated parameters should be near to the global minimum 
values to converge to the correct solution [12].

In order to isolate the contribution of each parameter, the simulation are performed first on a simplified scenario then its complexity is gradually increased. In the first analysis the effect of an observer on the Earth's surface is neglected, so a geocentric observer is used. At the same time the number of parameters to estimate is reduced accordingly with the chosen parametrization. In this case only semi-major axis, eccentricity, argument of perigee and the argument of latitude are estimated. Figure 2 shows the results obtained for this simplified scenario for an orbit with: $a=42164.173 \mathrm{~km}, e=0.6, i=30^{\circ}, \Omega=40^{\circ}, \omega=60^{\circ}$ and $T_{\text {osc }}=1^{\text {st }}$ observation.
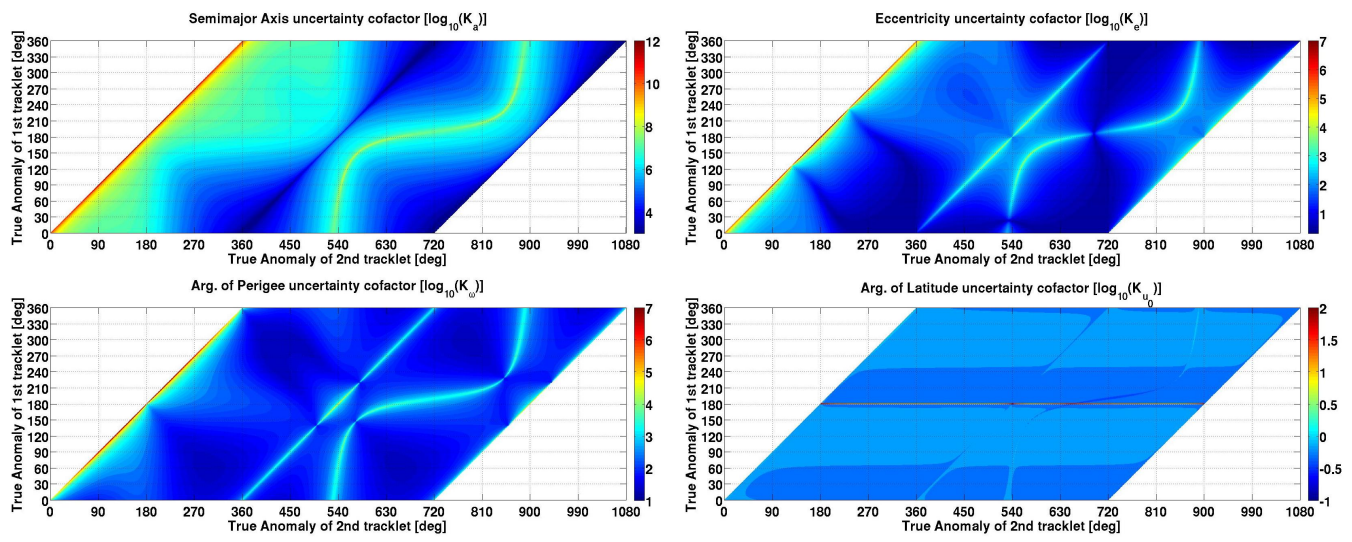

Figure 2: Uncertainty maps estimating only $a, e, \omega$ and $u_{0}$ with a geocentric observer.

Each point of Figure 2 shows the logarithm of the square root of the uncertainty of the estimated parameter as a function of the tracklets position. In particular, the position of the $1^{\text {st }}$ tracklet along the orbit can be read on the $y$-axis, while the position of the $2^{\text {nd }}$ is displayed on the $x$-axis. Both positions are expressed in terms of true anomaly. For completeness, we define uncertainty of the estimated parameter the square root of the relative term on the main diagonal of the covariance matrix divided by the a posteriori variance factor. Thus, to obtain the real value of the standard deviation, with the real unit of measurement, one has to multiply the value read in the map with the square root of the a posteriori variance factor according to Eq. (1). This factor being function of the residuals is expressed in square radians. Thus, the units of the semimajor axis map is $\mathrm{km} / \mathrm{rad}$, the one of the eccentricity is $1 / \mathrm{rad}$, while the ones for the angles (respectively $i$, $\Omega, \omega$ and $u_{0}$ ) are dimensionless.

In Figure 2 is already possible to notice two important characteristics: the first is given by the fact that the features in the maps are completely different between 
the first and the second orbital revolution, more precisely when the time interval between the tracklets is smaller or bigger than the orbital period. The second is the $S$-shaped feature appearing in the second revolution. The first effect is one of the consequences of the time dependency of some partial derivatives, while the $S$ shaped high uncertainty is occurring when the time interval between the tracklets is half an orbital period; both effects will be later discussed in details. Looking at the $S$-shaped high uncertainty it easy to see how for the eccentricity and the argument of perigee the features present some interruptions while for the semi-major axis the $S$ is continuous. Being this an elliptical orbit and the tracklets are acquired with a constant time sampling between observations, it is obvious that the arc of orbit covered by the tracklets is depending on the tracklet position. Furthermore, the length of the arc between the single observations is not constant. The mentioned effects provide information regarding the eccentricity and the position of the perigee. In fact if one pays attention to the interruptions of the $S$ for the eccentricity, these are occurring when the first tracklet is on the perigee and the second is at the apogee (and vice versa). This tracklets combination maximizes the difference between the lengths of the arcs covered by the tracklets. Regarding the interruptions of the $S$ for the argument of perigee, these are occurring when the tracklets are symmetric w.r.t. the line of the apsides. In this case two symmetric tracklets with the same length are used, but the distances between the observations within the tracklet are first increasing then decreasing (or vice versa) in the same way. This tells us if the apogee or the perigee is precisely in the middle of the arc defined by the two tracklets. If we pay attention to the position of the $S$-region, it is easy to see that is not precisely on the half period distance between the tracklets, this is even more evident if we look at the interruptions for the eccentricity. As we will see later, this is a second effect of the time dependency. For completeness also the uncertainty map for the argument of latitude $\left(u_{0}\right)$ is reported in Figure 2, this parameter will not be shown anymore on this paper because our interest is mainly focused on the geometric parameters. Furthermore, the uncertainties of $u_{0}$ are depending on the arbitrary time $T_{o s c}$. Finally, it is important to highlight two particular regions of the uncertainty maps: the first is constituted by the points lying on the diagonal line whose extremes have coordinates $\left[v_{2}=360, v_{1}=0\right]$, $\left[v_{2}=720, v_{1}=360\right]$ which defines all tracklets whose positions are separated by one (or more) orbital period in time; while the second consists of points lying on the diagonal line whose extremes have coordinates $\left[v_{2}=360, v_{1}=360\right]$, $\left[v_{2}=720, v_{1}=0\right]$ which defines all tracklets whose positions are symmetrical to the line of the apsides. Of course all the lines which are parallel to the before mentioned with a distance multiple of $360^{\circ}$ have the same meaning. 
Figure 3 shows the correlation maps obtained for the above mentioned scenario. The correlation index tells us how strong any two parameters are correlated. In particular if $|\rho|=1$ the two parameters are strongly correlated this means that any modification of one parameter will influence the other.

From Figure 3 is evident how the estimated parameters are strongly correlated. Some exceptions are present in these maps and coincide with the areas where the single parameters have their minimum uncertainty. For example if we look at the correlation between $a$ and $e$ or $a$ and $\omega$ the low correlation area are coincident with the "period" line. This result is clearly understandable because if we observe an object twice in the same position with an interval between tracklets of one period, the semi-major axis is well defined. Other areas with low correlation value are occurring in the graph $a$ vs $e$ when the first tracklet is on the perigee and the second on the apogee, while for the map $a$ vs $\omega$ low correlations are occurring for tracklets symmetric to the line of the nodes. It is also important to highlight that the maps in Figure 3 show the maximum correlation value in the $S$-area with the only exception given by the points where each parameter has its own uncertainty minimum.

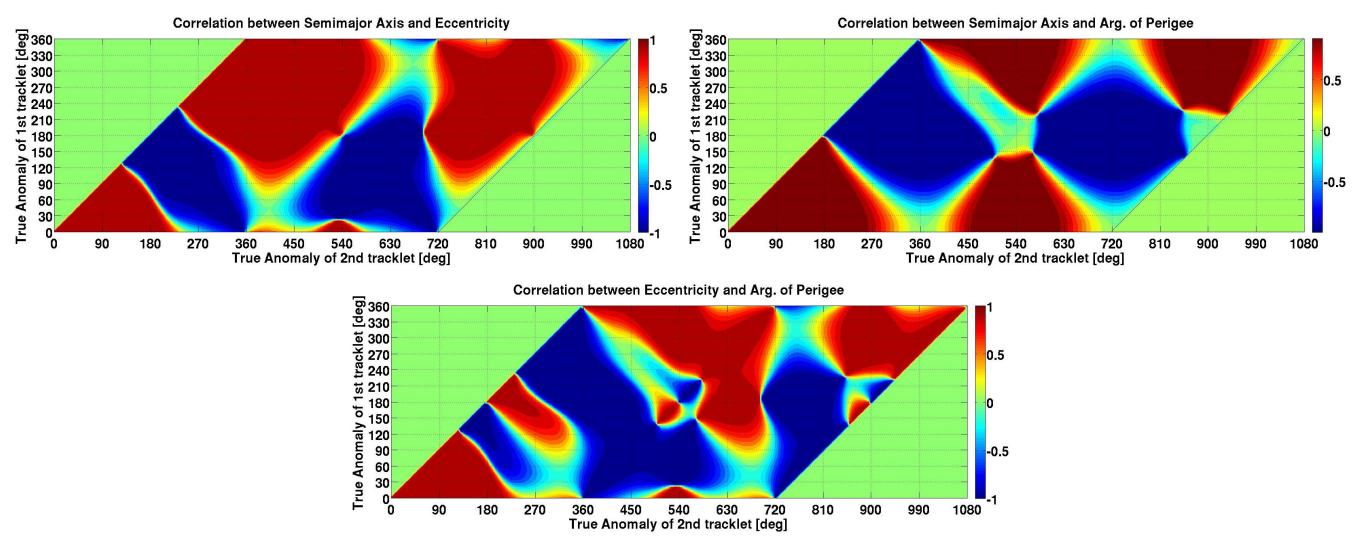

Figure 3: Correlation maps estimating only $a, e, \omega$ and $u_{0}$ with a geocentric observer. 


\subsection{The S-region}

In Figure 2 it is possible to see an $S$-shaped area of high uncertainty values very sharp for the semi-major axis, the eccentricity and the argument of perigee. As anticipated in the previous paragraph this area is occurring when the distance in time between the tracklets is half of an orbital period. Looking now at Figure 3 it is evident that in the $S$-area the LSQ is not able to distinguish among these three parameters, so in order to understand the cause of this $S$-shaped high-uncertainty area a simulation was performed fixing the argument of perigee at the level of the first design matrix $(A)$.
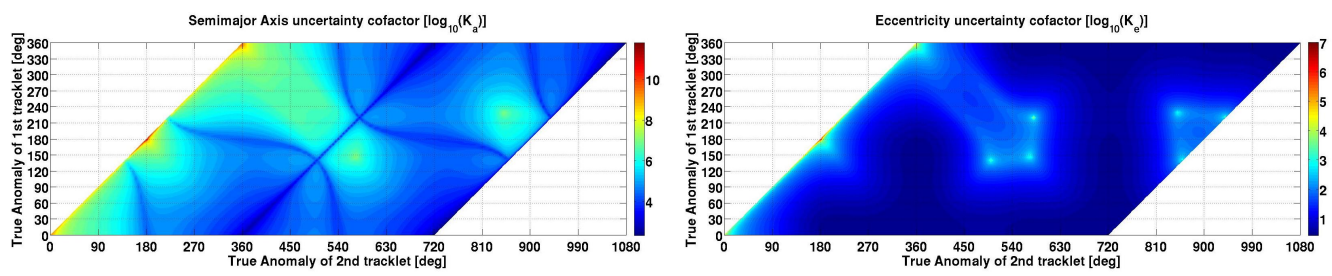

Figure 4: Uncertainty maps estimating only $a, e, \omega$ and $u_{0}$ with a geocentric observer but fixing $\omega$.

It is important to note that these results are not relevant for this study but at least they are helpful to find a possible explanation to the $S$-area. Looking at these results it is easy to see that from the $S$-region only two points are remaining, which coincide to the case where the position of the two tracklets is symmetric w.r.t. the line of the apsides with half a period distance. We think that the uncertainty on $a$ and $e$ is related to $\Delta t / \Delta \theta$ and this ratio is maximum for these particular points. We take a limit to $\Delta t=T / 2$ because each symmetric positions w.r.t. the line of the apsides with $\Delta t>T / 2$ is geometrically equivalent to the same positions after applying the $\bmod (\Delta t, T)$, where $T$ is the orbital period.

As seen before, if one estimates $a, e, \omega, u_{0}$ without fixing $\omega$, the above mentioned points disappear and instead of them we have the $S$-line containing all positions which are distant half a period in time. This is due to the fact that by varying $a, e$ and $\omega$ we can relate the generic situation of half a period distance between tracklets to the case of tracklets symmetric to the line of the apsides, just mentioned above.

\subsection{Influence of time interval}

As expected from the analysis of the partial derivatives and then confirmed by previous results, the time interval between the tracklets has a big influence on the 
uncertainties of the estimated parameters. This effect can be highlighted comparing the results in Figure 3 with those in Figure 5, which are obtained with a distance in time between tracklets contained within 49 and 50 orbital revolutions.
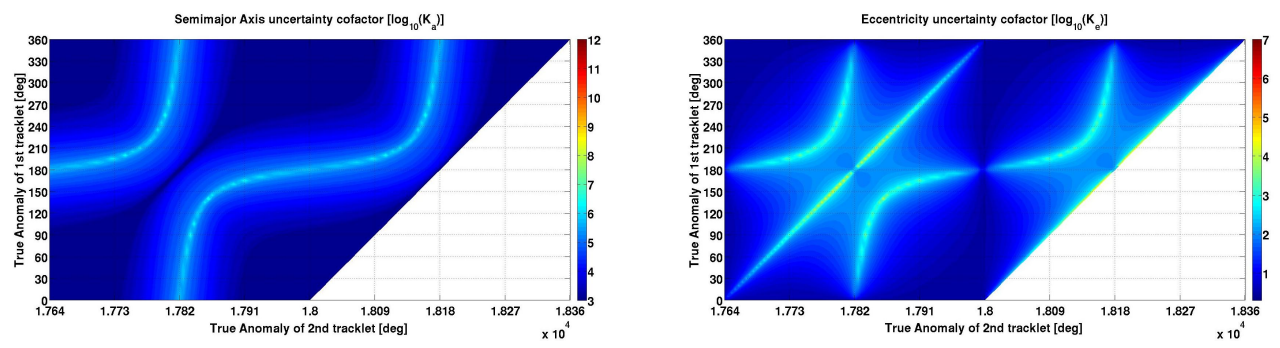

Figure 5: Uncertainty maps for $a$ and $e$ estimating only $a, e, \omega$ and $u_{0}$ with a geocentric observer with a maximum distance between tracklets of 50 orbital periods.

From this comparison it easy to see three main effects of the time distance between tracklets. Firstly, the $S$-shaped high uncertainty for $a, e$ and $\omega$ is clearly visible from the $2^{\text {nd }}$ period on. This does not mean that it is appearing only after one orbital revolution, but it is also present within the $1^{\text {st }}$ period; the only difference is that in this case, this feature is hidden under higher uncertainty values. Secondly, the average values of the uncertainties tend to decrease while the time interval between tracklets increases (specially for the semi-major axis). Thirdly, the $S$ shaped high uncertainty is now precisely passing on the area where the tracklets are separated, in time, by half of an orbital period. In Figure 6 it is possible to see how, specially for $a$, the average uncertainties are diminishing with time; and it is also clear how the half-period feature is hidden within high uncertainty values when the time interval between tracklets is less than one orbital revolution. A possible explanation for these phenomena is in the behavior of the partial derivatives. In fact, as seen before, the partial derivatives of position w.r.t. the semi-major axis contains a direct dependency from the time, in particular $d \vec{r} / d a \propto\left(t-T_{o s c}\right)$, while the others derivatives are periodic over the orbital period. Despite the fact that the time is increasing linearly, we think that these effects are due to the order of magnitude of the time difference between the tracklets, which is growing from 0 up to $10^{4}$ seconds within the $1^{\text {st }}$ orbital period, then from $10^{4}$ to $10^{5}$ in the $2^{\text {nd }}$, and it will reach $10^{6}$ only after the 11 th revolution.

\subsection{Estimating also $i$ and $\Omega$}

Once we understood the main features characterizing $a, e, \omega$ and $u_{0}$, the simulation was repeated including in the estimation process also the parameters which describe the orientation of the orbital plane in the space ( $i$ and $\Omega$ ). Figure 7 shows the 


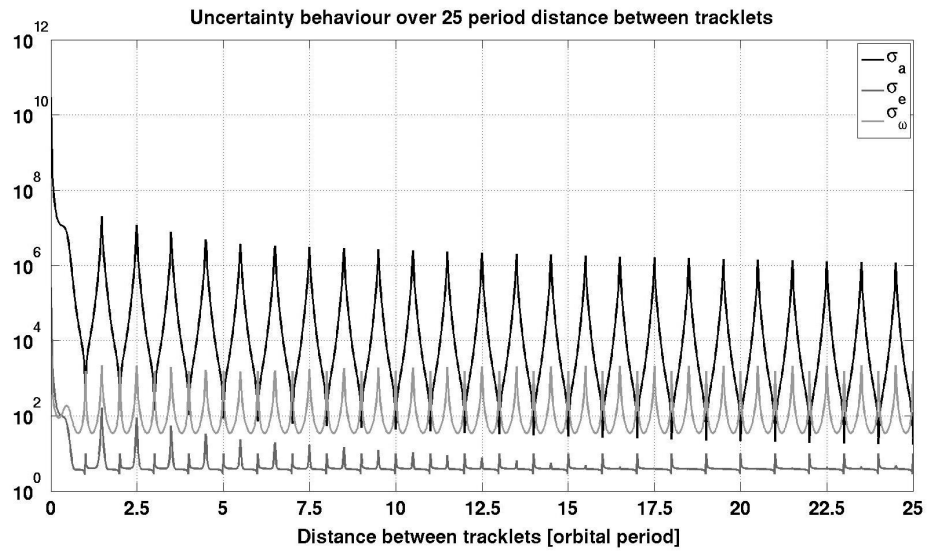

Figure 6: Behavior of the uncertainty of $a, e$ and $\omega$ obtained with the $1^{s t}$ tracklet on the perigee with a varying time interval of the $2^{\text {nd }}$ up to 25 orbital revolutions.

uncertainty maps obtained for the same orbit analyzed before $(a=42164.173 \mathrm{~km}$, $e=0.6, i=30^{\circ}, \Omega=40^{\circ}, \omega=60^{\circ}$ and $T_{o s c}=1^{\text {st }}$ obs.) with a geocentric observer.
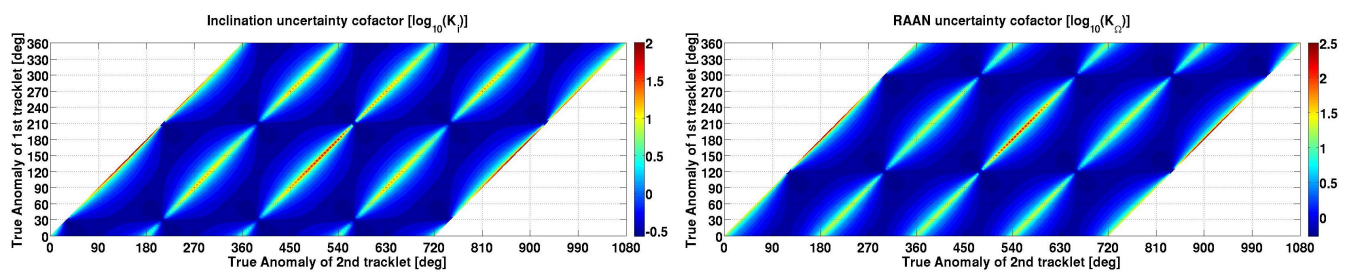

Figure 7: Uncertainty maps for $i$ and $\Omega$ estimating all 6 parameters with a geocentric observer.

Figure 7 shows how the uncertainty maps of $i$ and $\Omega$ are characterized only by one main feature of high uncertainty values which includes all the positions with a difference in time of one (or more) orbital period and which are separated by $180^{\circ}$ in true anomaly. These high uncertainty areas can be explained considering the fact that the orientation of the orbital plane in the space is described by the direction of the angular momentum. At the same time this direction is also defined by the cross product of the directions of two position vectors. The cross product is undefined if the unit vectors involved are parallel or aligned which is precisely the case described just before. Each of these features present two interruptions. To explain them it is necessary to have a look at the orbital parameters, in particular to $\omega$, which in this case is equal to $60^{\circ}$. In this case the line of the nodes intersects the orbit when the true anomaly is equal to $120^{\circ}$ and $300^{\circ}$. Being the observer always within the orbital plane, the information regarding $\Omega$ is maximized if both 
observations are performed on the line of the nodes. The information about the inclination is maximized if the observations are performed at $90^{\circ}$ of distance from the line of the nodes, in this case when $v$ of the $1^{\text {st }}$ tracklet is equal to $30^{\circ}$ and for the second tracklet $v=210^{\circ}$, or vice versa.

\section{Results with a real observer}

Until now, the results shown were obtained using an unreal observer located in the center of the Earth. In the next section, the influence of a real observer on the Earth's surface is analyzed. The Figure 8 shows the scenarios used to isolate the contributions given by a topocentric observer. Two "standard" orbits are used to carry out this study and the results obtained using different observer displaced in longitude or in latitude are compared. The used orbits have the following orbital elements: $a=42164.173 \mathrm{~km}, e=0.6, i=1^{\circ}$ or $60^{\circ}, \Omega=0^{\circ}, \omega=0^{\circ}$ and $T_{\text {osc }}$ coincides with the time of first observation. As for the case of the geocentric observer, the simulations are performed analyzing all possible combinations between tracklets positions. The only difference is the observer position. In particular, the initial position of the observer is kept constant for the different $1^{\text {st }}$ tracklet postions while for the second tracklet, the position of the observer is rotated accordingly with the time interval between tracklets and the Earth's angular velocity. The initial positions chosen for these simulations are shown in Table 1. It is important to note that being a geometrical study the real orientation of the Earth in the space is neglected. To better understand the results we set up the orbits so that their line of nodes and their line of the apsides are coincident with the $x$-axis of the generic inertial system; and the Greenwich meridian is also aligned with the same axis at time $t=0$.

\begin{tabular}{lccc} 
& Long. [deg] & Lat. $[\mathbf{d e g}]$ & Alt. $[\mathrm{m}]$ \\
\hline Reference Observer & 0 & 0 & 0 \\
Longitude-displaced observer & $60^{\circ}$ East & 0 & 0 \\
Latitude-displaced observer & 0 & $50^{\circ}$ North & 0 \\
\hline
\end{tabular}

Table 1: Observer geodetic coordinates.

\subsection{Influence of longitude}

On the first attempt, to isolate the contribution given only by the position of the observer, the simulation are performed using an almost equatorial orbit, to avoid 


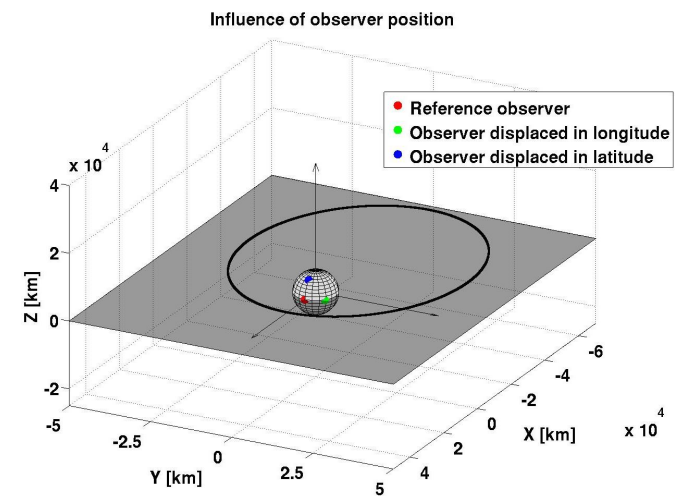

(a) Different observer positions with an equatorial orbit

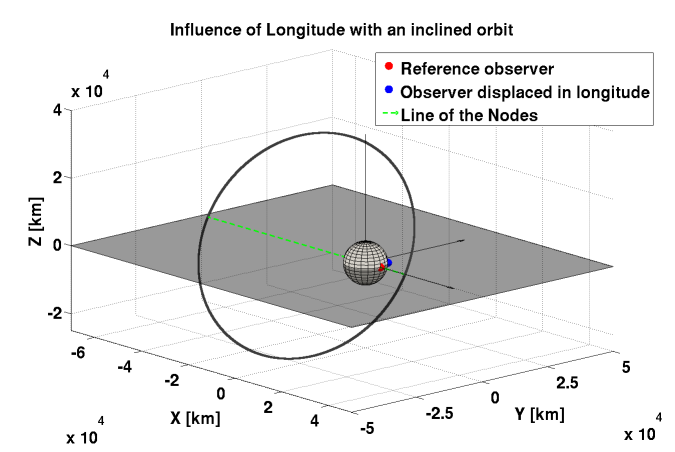

(b) Different observer positions with an inclined orbit

Figure 8: Scenarios used to evaluate the influence of a real observer.

the singularity of the used parametrization an inclination of $1^{\circ}$ is used. In fact for $i=0^{\circ}$ it is not possible to distinguish anymore between $\Omega$ and $\omega$. The results obtained with a longitude displacement of the observer with an equatorial orbit are analyzed and no significant effects can be seen. A "more" complicated scenario in which $i=60^{\circ}$ is used to evaluate the effects of a longitude-displaced observer. The simulation are then repeated using the scenario described in Figure 8b, where the "Reference" observer, at the time of $1^{\text {st }}$ tracklet, is within the orbital plane precisely on the line of the nodes, which in this case coincides also with the line of the apsides. Being a geosynchronous orbit, the observer will be again within the orbital plane every half of a revolution. For the observer displaced in longitude the $1^{\text {st }}$ tracklet is acquired always outside of the orbital plane. The observer will be in the orbital plane only if the distance in time between the tracklets is equal to $1 / 3$ or $5 / 6$ of the orbital period. Figure 9 shows the results of this simulation and shows the comparison between only two parameters $a$ and $i$. The just mentioned figure (Figure 9) shows the comparison of the uncertainties obtained for a time distance between tracklets included from 4 and 5 orbital revolutions; it was decided to show these results to take into account the shift of the main features due to the time dependency of the derivatives (see paragraph 3.2). Comparing the latter results with those obtained in the case of an equatorial orbit we can already conclude that the relative position of the observer w.r.t. the orbital plane has a huge influence on the results, in particular it is important to distinguish if the observer is always, systematically or just occasionally within the orbital plane. Figure 9 
shows the comparison between results obtained with an observer systematically inside the orbital plane w.r.t. that obtained by an observer just occasionally inside. The Figures $9 \mathrm{a}$ and $9 \mathrm{~b}$ show some common features as: the minimum uncertainty area on the period line for $a$, the $S$-area with high uncertainties, the high uncertainties for tracklets whose angular distance is $180^{\circ}$ for $i$. Focusing now on the uncertainty maps for $a$, it is possible to see few differences and one new feature. The main difference is in the uncertainty values in the $S$-area which are smaller if the observer is just occasionally within the orbital plane. The new feature that is not so sharp in this case, but will be more evident in the next paragraph, is constituted by a minimum value line for $a$ in the case where the tracklets have an angular distance of $180^{\circ}$. These two effects are due to the fact that observing from outside the orbital plane one is able to estimate the distance to the object.

Comparing the two inclination maps, it is easy to see that they are pretty different, in particular the one in Figure 9a shows two horizontal lines with low uncertainty values and an $S$-shaped low uncertainty area. The reason for this is that the two horizontal lines are occurring precisely when the true anomaly of the $1^{\text {st }}$ tracklet is $90^{\circ}$ or $270^{\circ}$; remembering that $\omega=0^{\circ}$ these two are the points of the orbit with the maximum angular distance from the line of the nodes. In the figure only the horizontal lines are present because they are relative to the $1^{\text {st }}$ tracklet which is always acquired with the observer within the orbital plane; while if the $2^{\text {nd }}$ tracklet is in the same positions the observer is outside the plane reducing the strength of this information. Regarding the $S$-area, it contains all the observations performed by the observer within the orbital plane.

Looking now at the inclination map of Figure 9b, the just mentioned features are not present anymore but only some minimum point-like areas can be observed. These areas are given by a compromise between distance of the observation from the line of the nodes and position of the observer w.r.t. the orbital plane. These higher average uncertainty values of this figure also mean that to determine $i$ and $\Omega$ is better to have an observer within the orbital plane. It was decided not to show the maps relative to $e, \omega$ and $\Omega$ because we can extract the same conclusions just reported for $a$ and $i$. 

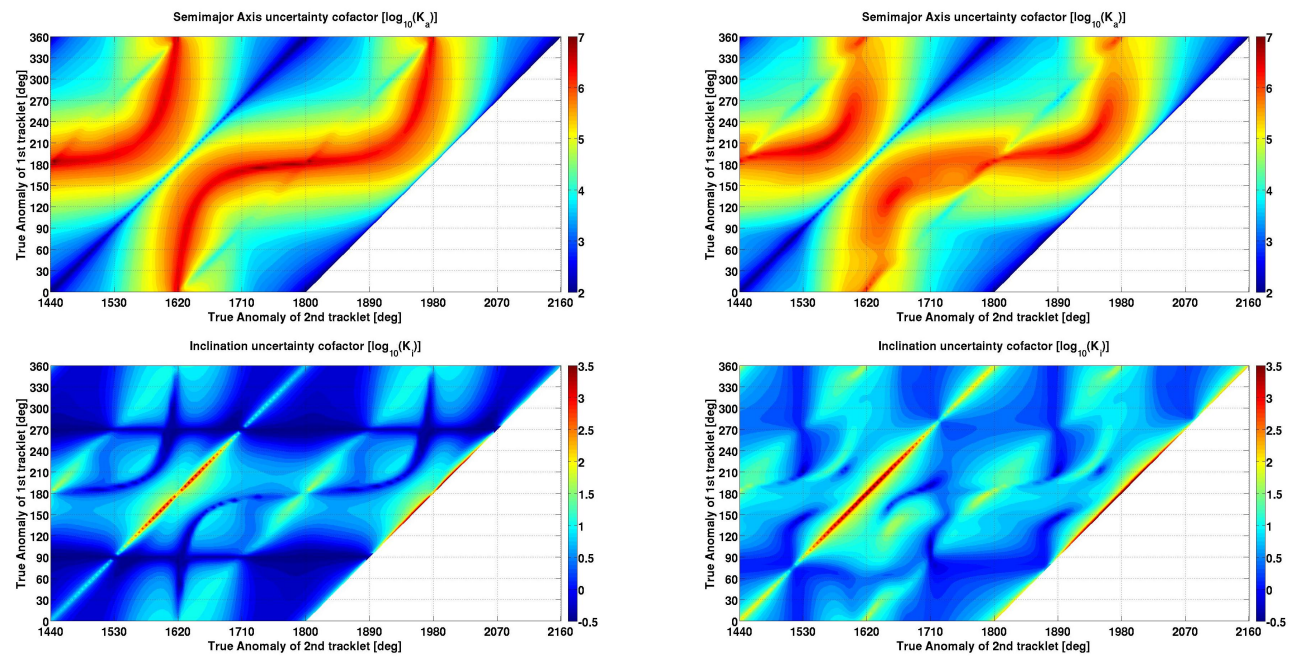

(a) Observer every half a period within the orbital plane

(b) Observer occasionally within the orbital plane

Figure 9: Comparison between uncertainty maps obtained for an inclined orbit with longitudedisplaced observer.

\subsection{Influence of Latitude}

To confirm the importance of the position of the observer w.r.t. the orbital plane, the results obtained in the case where the observer is always inside the orbital plane with the case where it is always outside are compared. The geosynchronous equatorial orbit is again used with an equatorial observer and the results are compared with those obtained with the same orbit but the observer was displaced by $50^{\circ}$ in latitude (see Figure 8a). Figure 10 shows the uncertainty maps for $a, i$ and $\omega$ obtained with the equatorial observer and the ones obtained with a latitudedisplaced observer (respectively in Figure 10a and 10b). As for Figure 9, also in this case, due to the effects of the time dependency, it was decided to show the results obtained from a time distance between tracklets of 4, 5 orbital revolutions. Comparing the uncertainty maps for $a$ it is clearly visible how the intensity of the $S$-area is strongly reduced by an observer outside the orbital plane; on the map of $e$ the same effect is noticeable. As for the previous paragraph a diagonal line with low uncertainty values is appearing when the $v_{2}-v_{1}=180^{\circ}$; this effect can be explained by the fact that an orbit is a section of a cone. If one is observing the orbit from the apex of the cone (or from a circular section of the cone) and one is able to estimate the distances, exploiting the information within the tracklets, it is 
easier to determine the semi-major axis and the eccentricity.

Comparing now the maps for the inclination we can see how the main features are kept. There is only a small reduction of the diagonal line with tracklets separated by $180^{\circ}$. The most important difference is that the average uncertainty values in the Figure 10b remarkably increased. This is well understandable because the inclination can be understood measuring the distance of the object from the equatorial plane. Having a relatively small distance, given by $1^{\circ}$ of inclination, this can be better measured if the observer is inside the orbital plane. One interesting result is the differences between the maps relative to the argument of perigee. In the case in Figure 10a the classical $S$-shaped area is present, it is then important to notice that also a high uncertainty diagonal line is present for tracklets with angular distance of $180^{\circ}$. This feature is characteristic of $\Omega$ but is present also on $\omega$ because of the small inclination value. For $i \rightarrow 0$ it is difficult to define the line of the nodes and being the argument of perigee defined w.r.t. this line all the uncertainties of $\Omega$ are transferred also to $\omega$. The most interesting feature for the map of $\omega$ in Figure 10b is given by the fact that the $S$-area contains minimum uncertainty values. This can be explained by the fact that looking from outside the orbital plane we have an improvement given by the capabilities to estimate the distances. This is followed by an improvement also for the eccentricity estimation, and being able to estimate these two quantities, one is also able to decorrelate them from $\omega$. Finally, the increase of the average uncertainty values can be explained by the fact that looking from outside the orbital plane it is more difficult to determine precisely the distance between observations within a tracklet.

In conclusion, is it better to observe within or outside the orbital plane? There is not a unique answer to this question as we have just seen: the observer inside the orbital plane is gaining more information about the orientation of the orbital plane in the space, amplifying the effect given by the distance of the observations from the line of the nodes and reducing the average uncertainty values. Vice versa, observing from outside the orbital plane gives us information about distances that reduce the average error on $a$, strongly reduce the $S$-area for $a, e$ and $\omega$, and helps us to decorrelate $\omega$ from $a$ and $e$.

\subsection{Influence of orbital parameters}

The influence of $u_{0}$ was studied: modifying $u_{0}$ means that at $T_{\text {osc }}$ the object is at a different place of the orbit. This analysis showed that this parameter is affecting only the map of the argument of latitude while is not influencing at all the other geometric parameters.

The $\omega$ parameter describes the orientation of the orbit within the orbital plane, 

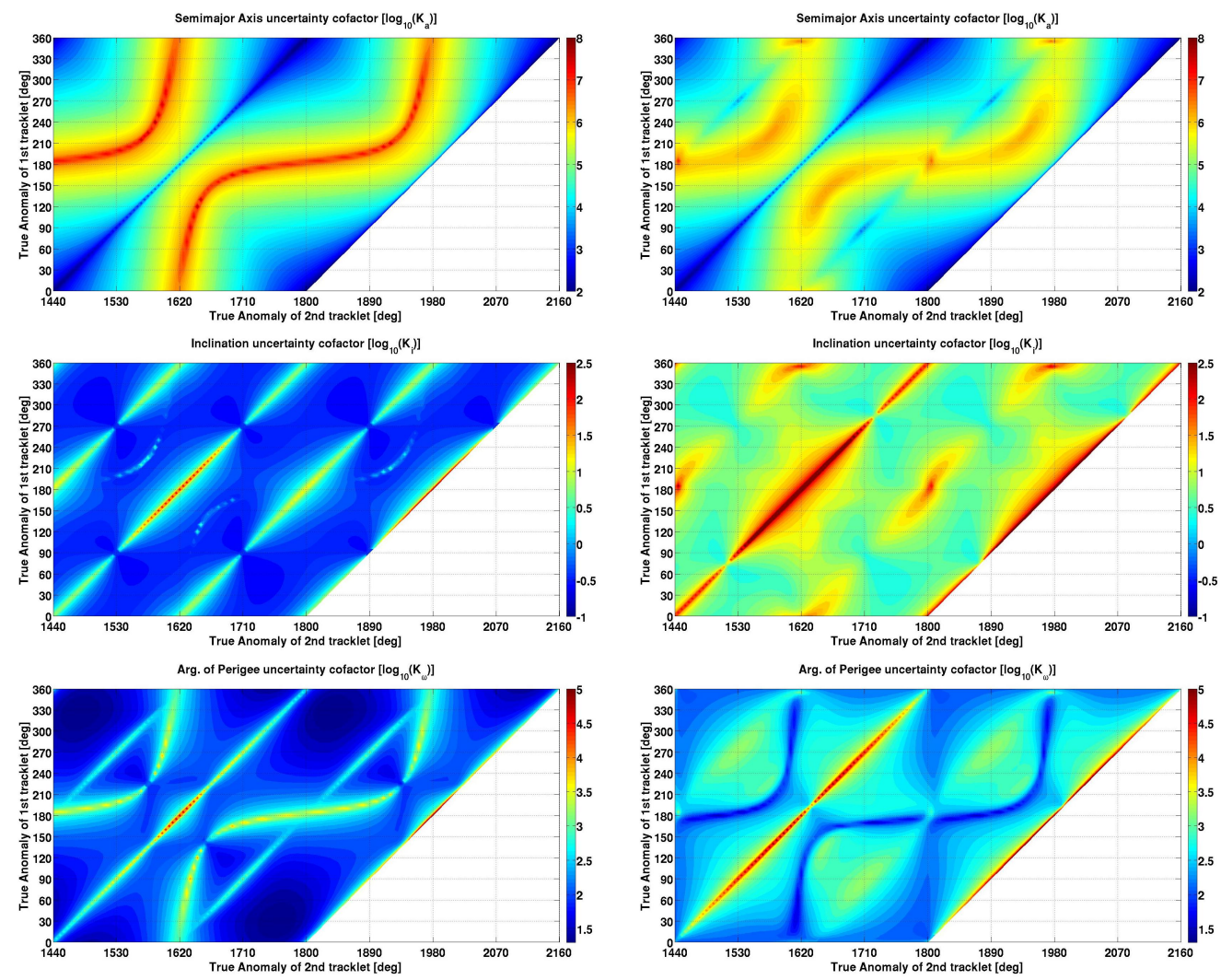

(a) Equatorial observer

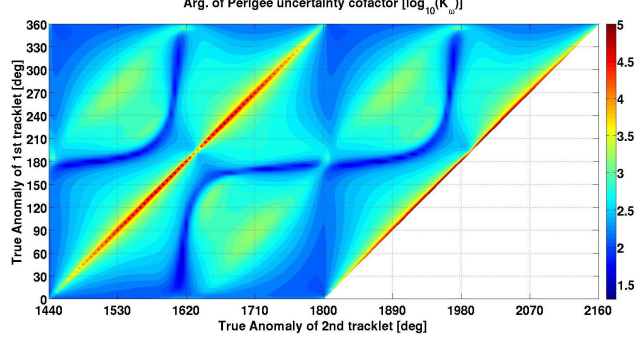

(b) Latitude-displaced observer

Figure 10: Comparisons between uncertainty maps obtained for an equatorial orbit with latitudedisplaced observer. 
changing it, means changing the intersection points of the orbit with the equatorial plane producing only a translations of the minimum horizontal lines seen in Figure 7.

Repeating the simulations changing the position of the line of the nodes in the space $(\Omega)$ produces the same effect of a displacement in longitude of the observer changing the relative position of the observer w.r.t. the orbital plane. In particular the observer will be inside or outside the orbital plane for different time distances between the tracklets.

One of the most interesting parameter is the inclination $(i)$, as anticipated before, the main effects are given when $i$ tends to 0 . If $i=0 \Longrightarrow \Omega$ is not defined, the main effects of this problem are shown in the map for $\omega$ in Figure 10a. Being $\omega$ defined w.r.t. the line of the nodes, it will show some characteristic features of $\Omega$. Additionally the closer is $i$ to 0 the fainter will be the characteristic $S$-area of $\omega$. It must be taken into account also that $u_{0}=\omega+v_{0}$, for this reason, the main features of $\Omega$ will be transferred as well to the argument of latitude.

The eccentricity is the main responsible for the $S$-area, in particular if $e$ grows the $S$ tends to have the shape of two hyperbolas, while if $e \rightarrow 0$ (for circular orbit) the $S$ becomes a diagonal line for tracklets whose angular distance is $180^{\circ}$.

The last important effect is given by the variations of the semi-major axis which is related to two parameters: the parallax due to the Earth and the length of the arc covered by a tracklet. Increasing the distance of the object from the Earth, that means increasing $a$ of the orbit, the beneficial effect of the Earth parallax and of the information within the tracklet are strongly reduced. If the object is enough distant from the Earth the effect given by the Earth's parallax and the fact that the observer is inside or outside the orbital plane is strongly reduced and the features of the maps become similar to those obtained for a geocentric observer. During all simulations the time interval between the measurements within a tracklet is kept constant; this leads to a decrease of the arc covered by the tracklet if the semimajor axis increase. The main consequence of a shorter arc of a tracklet is an increase of the average uncertainty values especially for $a, e$ and $\omega$ which exploit the information given by the relative distance between the measurements.

\subsection{Influence of Earth's rotation}

Another aspect that should be taken into account during the maps analysis is the rotation period of the Earth. In particular the ratio between the orbital period and the Earth rotation is important because it will determine when an observer is within or outside the orbital plane. Figure 11 shows the results obtained for the uncertainty maps of $a$ for two orbits which have the same values of $e, i, \Omega, \omega$ 
and $u_{0}$, but different $a$ values; in particular in Figure 11a $a=20270.404 \mathrm{~km}$ and in Figure $11 \mathrm{~b} a=42164.173 \mathrm{~km}$. These two values of $a$ were chosen in order to have, in the first case, three complete revolution of the satellite and, in the second case, one within the Earth's rotation period. This choice allowed us to have an observer within the orbital plane respectively every 1.5 and 0.5 orbital periods. This experiments confirms the fact that the $S$-area is characteristic of observations performed within the orbital plane; as shown in Figure 11a, the $S$ appears only when the distance between tracklets is 1.5 or 4.5 orbit revolutions.

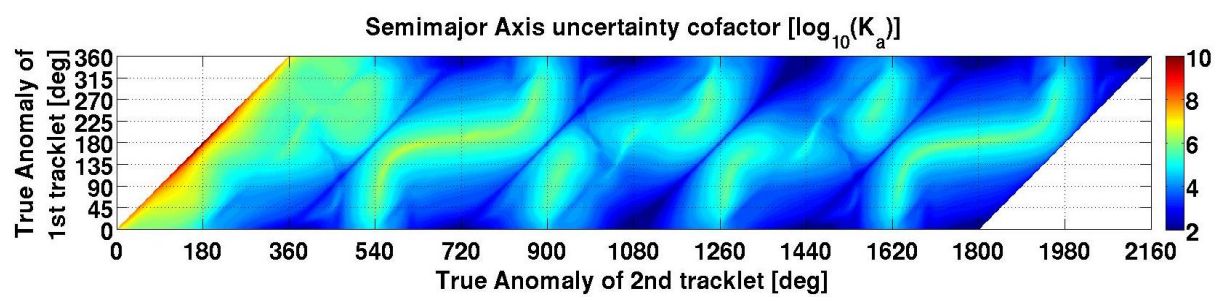

(a) $a=20270.404 \mathrm{~km}, 3 \mathrm{rev} /$ day

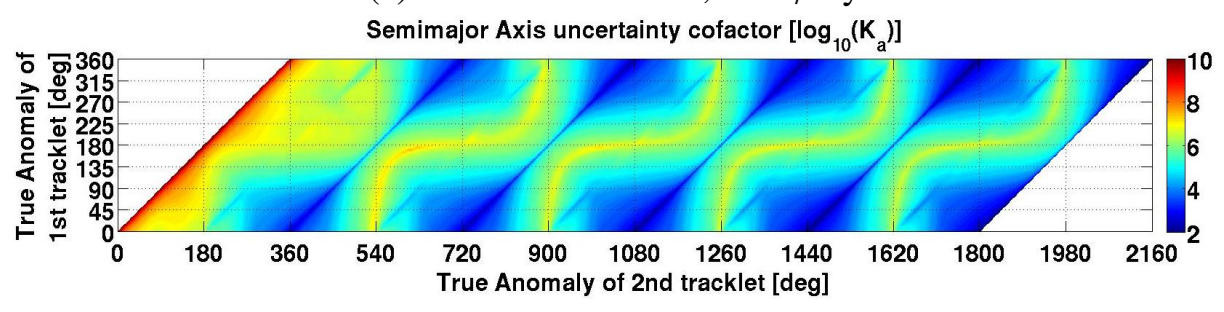

(b) $a=42164.173 \mathrm{~km}, 1 \mathrm{rev} /$ day

Figure 11: Influence of Earth's rotation for the semi-major axis uncertainty map.

\subsection{Visibility conditions}

The current maps show also some impossible combinations of observations due to the visibility limits; for example if we consider the scenario described at the beginning of Chapter 4 where the perigee of the orbit is just at the zenith of the observer at time $t=0$, it is obvious that all the $1^{\text {st }}$ tracklet positions which are around the apogee are impossible to see because one should be able to look through the Earth. Are there some "forbidden" regions that is not possible to see in any case? How will the daylight time influence our maps? In fact it is always possible to find an orbit whose parameters fulfill my visibility criteria. For example, the results of two simulations were compared: in the first the orbital parameters described in Chapter 4 were used, and in the second the same orbital elements were kept but 
$\omega$ was set to $180^{\circ}$, so that the apogee of the orbit was at the zenith at time $t=0$. This comparison showed that the features presents in the maps are precisely the same, the only difference are the uncertainty values which are slightly different due to the different distances of the object from the observer. Then, it is possible to conclude that the maps do not have forbidden regions but to apply them it is necessary to use a mask for the elevation limits and another one for the nighttime.

\section{Real cases}

Until now only a theoretical study was performed to highlight the influence of each single parameter on the accuracy of an orbit determination/improvement process. The main features are now known and it is also known the effect of the observer position. The next natural step is to apply this method to a real scenario characterized by a real orbit with a real observer. For simplicity the simulation are carried out using a generic inertial system of reference. The following paragraph will show the results obtained for two orbits which are largely populated by satellite and then by space debris: the geostationary orbit (GEO) and the geostationary transfer orbit (GTO).

\subsection{GEO}

The orbital parameters used for this simulations are: $a=42164.173 \mathrm{~km}, e=$ $0.0005, i=0.1^{\circ}, \Omega=270^{\circ}, \omega=0^{\circ}$ and $T_{o s c}=1^{s t}$ observation. For the observer's position the Zimmerwald observatory is used whose geodetic coordinates are: $46.8772^{\circ}$ North, $7.4652^{\circ}$ East and $951.2 \mathrm{~m}$ altitude. A maximum distance of 2 orbital revolutions between the two tracklets is allowed in this simulation.

Figure 12 shows that, being an almost circular orbit, the $S$-area for $a, e$ and $\omega$ is now a diagonal line that is not yet precisely coincident with that of $180^{\circ}$ angular distance between tracklets because of the time dependency shown in paragraph 3.2. In these maps is also present the diagonal line for tracklets whose distance is $180^{\circ}$ given by the fact that the observer is always outside the orbital plane. Looking at the uncertainty maps for $i$ and $\Omega$, the high-uncertainty diagonal lines for tracklets separated by $180^{\circ}$ and one orbital revolution are clearly visible. It is interesting to notice that the fact that the $180^{\circ}$ tracklets separation is not the best configuration of observation, it is in any case better than observing twice on the same place (for $i$ and $\Omega$ ). Finally, other consequences of the small eccentricity values can be seen in the average high uncertainties for $\omega$. In this case, despite the small inclination value, the argument of perigee keeps its main features while the uncertainties of $\Omega$ are influencing $u_{0}$ which is completely correlated with it. 

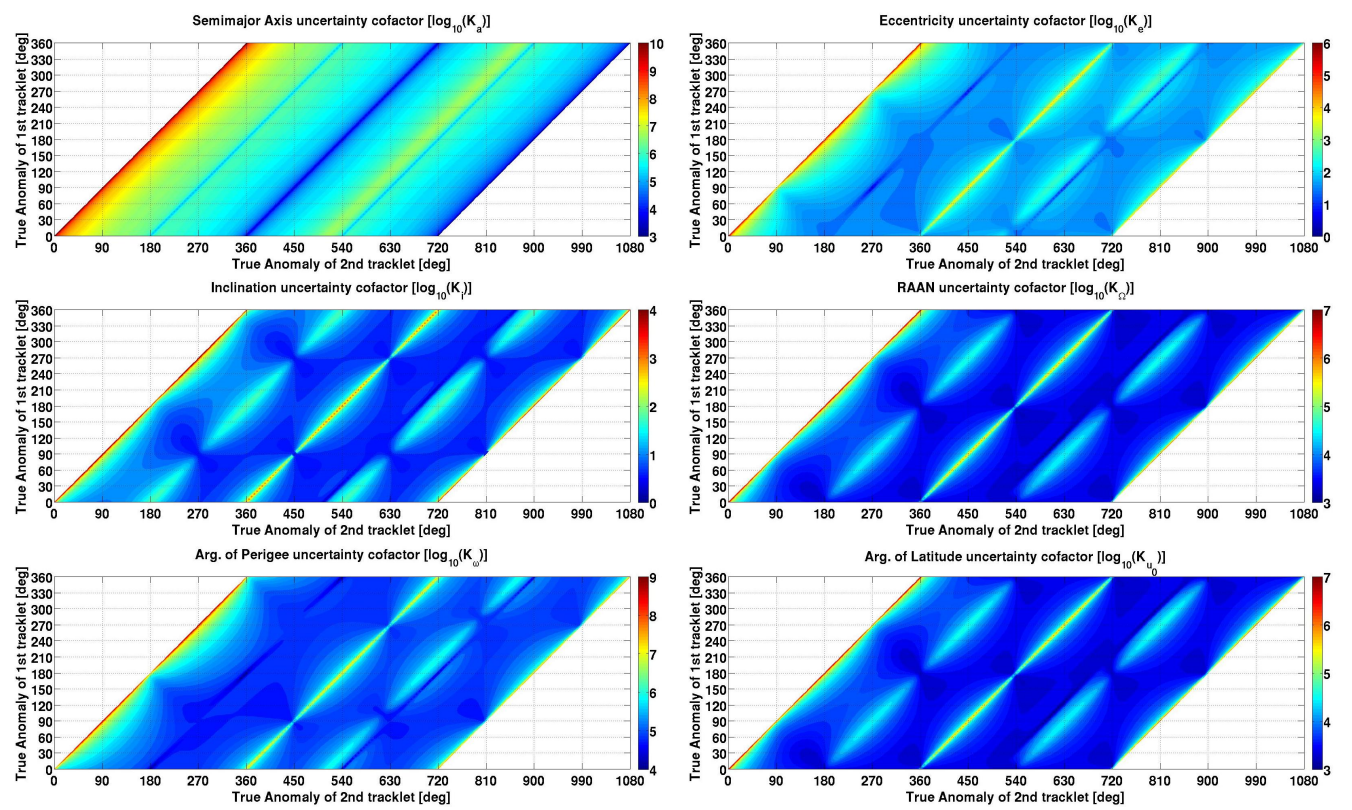

Figure 12: Uncertainty maps for GEO orbit observed from Zimmerwald.

\section{2. $G T O$}

The orbital parameters used for this simulations are: $a=24409.4 \mathrm{~km}, e=0.7287$, $i=6^{\circ}, \Omega=0^{\circ}, \omega=226^{\circ}$ and $T_{\text {osc }}=1^{\text {st }}$ observation; for the observer the Zimmerwald observatory is selected and a maximum distance of 2 orbital revolutions between the two tracklets is allowed. Figure 13 shows the uncertainty maps obtained for the GTO case. Although the results are difficult to interpret, it is still possible to see some main features as: the decrease of the values for the semi-major axis when the distance in time between tracklets increases, the $S$-area almost disappeared for $e$ and $\omega$ while for $a$ it is strongly reduced, and the presence of the minimum uncertainty diagonal line for tracklets separated by $180^{\circ}$. The latter two effects are both caused by the fact that the observer is always outside the orbital plane. Looking at the uncertainty maps for $i$ and $\Omega$ it is possible to see how the diagonal lines for tracklets separated by $180^{\circ}$, even if still present, are strongly reduced. The high uncertainty area remains when the two tracklets are both close to the apogee. In these two maps it is also possible to see two horizontal lines of low uncertainty values, respectively when the $1^{\text {st }}$ tracklet is between $30^{\circ}$ and $60^{\circ}$ for $i$ and between $300^{\circ}$ and $330^{\circ}$ for $\Omega$. Knowing that $\omega=226^{\circ}$ these regions coincide with the maximum distance from the line of the nodes, and the position of the line of the nodes, respectively. Finally it is important to notice how, 
due to small inclination value, the feature of $\Omega$ are contaminating the maps of the argument of perigee and the argument of latitude.
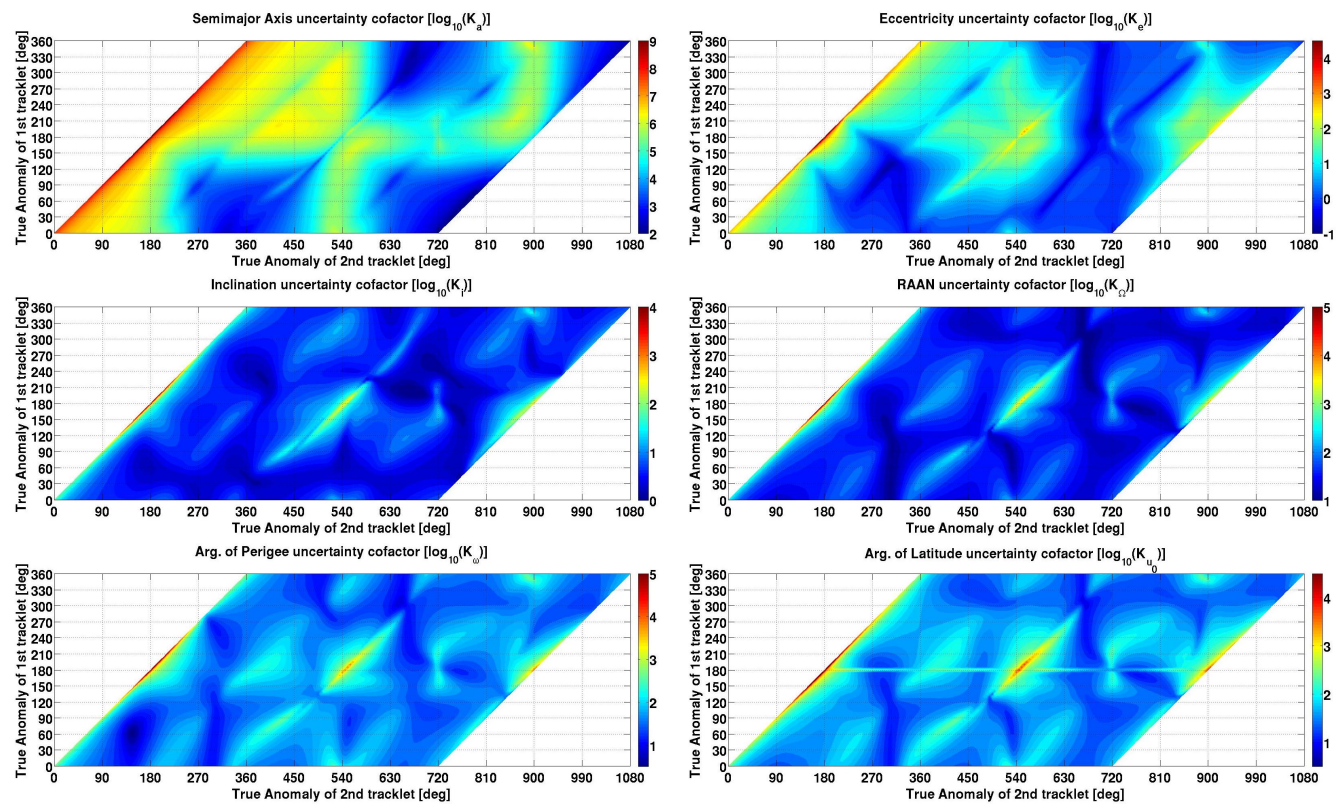

Figure 13: Uncertainty maps for GTO orbit observed from Zimmerwald.

\section{Conclusions and future works}

A fundamental step in space debris research consists in improving the knowledge of the orbit of observed objects. Since this activity is usually performed with telescopes, which are weather dependent, and due to the high number of objects that have to be observed, it is necessary to optimize the time available for observations, being it for surveys or follow-ups. In this work, a study was presented to highlight the dependency of the accuracy of the results achievable from an orbit determination on the object-observer relative geometry. This study was performed analyzing the covariance matrix obtained from the simulation of a LSQ adjustment process. In particular, we simulated an orbit determination/improvement problem, in which we evaluate the consequences given by two series of observations on the accuracies of the estimated parameters. The relative position of the two series of observations were chosen in order to cover all possible combinations of tracklets positions. The results of the simulations allowed us to create an uncertainty map for each estimated parameter, which shows the uncertainties as a function of the 
position of the first series of observations and the separation in time between the series. At the beginning, this analysis was performed for a simplified scenario without estimating the complete set of parameters using an observer on the center of the Earth. Such simplification of the problem allowed us to better understand what kind of results are achievable, the influence of the time interval between tracklets and what the limitations of the parametrization are. First, we introduced the parameters which describe the orientation of the orbital plane in the space. Subsequently an observer on the Earth's surface was introduced in the LSQ process. This study was repeated for different observer positions and different kinds of orbit. This study allowed us to understand the main factors which are influencing the accuracies of the estimated parameters as: the time interval between tracklets, the inclination of the orbit, the distance from the line of the nodes, the angular distance between tracklets, the influence of the position of the observer w.r.t. the orbital plane, the distance of the object in terms of Earth's parallax and arc-length of the tracklets, and finally the difference between orbital period and sidereal day. It is important to notice that it is not possible to obtain a unique conclusion from the uncertainty maps, but these can be useful depending on the needs of the users. In fact, having an a priori knowledge of the orbit or of the orbital region that one wants to scan (respectively in the case of follow-up and survey), the maps show where to observe in order to minimize the uncertainty on certain parameters or to obtain the best initial orbit.

The proposed method can be used to study what kind of improvement can be obtained introducing a second observer or a different observable, like ranges, in an orbit determination problem. Finally the study can be applied to more general situations, where more than two series of observations are available in order to optimize the survey/follow-up strategy.

\section{Acknowledgment}

The first author would like to thank the Swiss National Science Foundation for providing the funding that supported this work (SNSF Grant 200020 157062).

\section{References}

[1] ESA Website, Clean Space page, 2015. http : //www.esa.int/Our_Activities/S pace_Engineering_Technology/ Clean_S pace/How_many_space_debris_objects_are_currently_in_orbit 
[2] United Nations, Office for Outer Space Affairs. Space Debris Mitigation Guidelines of the Committee on the Peaceful Uses of Outer Space. Vienna, Austria, 2010.

[3] United Nations, Office for Outer Space Affairs. Technical Report on Space Debris. A/AC.105/720, United Nations Publication Sales No. E.99.I.17, ISBN 92-1-100813-1, United Nations, New York, USA, 1999.

[4] National Research Council (U.S.), Committee on Space Debris. Orbital debris: a technical assessment. National Academy Press, ISBN 0-309-051258, Washington D.C., USA, 1995.

[5] Schildknecht, T., Herzog, J., Vananti, A., Ploner, M., Fletcher, E. Coordinated Optical GEO Survey for European SSA Precursor Services. Proceedings of AMOS Conference, Maui, Hawaii, 2013.

[6] Schildknecht, T., Vananti, A., Hinze, A., Herzog, J., Ploner, M. Long-term evolution of high area-to-mass ratio objects in different orbital regions. Proceedings of AMOS Conference, Maui, Hawaii, 2012.

[7] Vallado, D., Agapov, V. Orbit Determination Results from Optical Measurements. Proceedings of AAS/AIAA Astrodynamics Specialist Conference, Toronto, Canada, 2010.

[8] Schildknecht, T., Herzog, J., Hinze, A., Vananti, A., Ploner, M. AIUB efforts to survey, track, and characterize small-size objects at high altitudes. Proceedings of DLRK Conference, Berlin, Germany, 2012.

[9] Cowardin H., Seitzer P., Abercromby K., Barker E., Buckalew B., Cardona T., Krisko P., Lederer S. Observations of Titan IIIC Transtage fragmentation debris. Proceedings of AMOS Conference, Maui, Hawaii, 2013.

[10] Silha, J., Schildknecht, T., Hinze, A., Vananti, A. Additional optical surveys for space debris on highly eccentric and inclined MEO orbits. Proceedings of 65th International Astronautical Congress, Toronto, Canada, 2014.

[11] Beutler, G. Methods of Celestial Mechanics, Vol I/II. Springer-Verlag, ISBN:978-3540407492/ISBN:978-3540407508, Berlin, Germany, 2005.

[12] Vallado, D. A. Fundamentals of Astrodynamics and Applications - 4th Ed. Microcosm Press, ISBN:978-1881883180, 2013. 